\title{
Cognitive changes as a result of a single exposure to placebo
}

\author{
Debbie L. Morton $^{\mathrm{a}, *}$, Christopher A. Brown ${ }^{\mathrm{a}}$, Alison Watson ${ }^{\mathrm{a}}$, Wael El-Deredy ${ }^{\mathrm{b}}$, Anthony K.P. Jones ${ }^{\mathrm{a}}$ \\ a Human Pain Research Group, University of Manchester Rheumatic Diseases Centre, Salford Royal Hospital, Salford, Manchester M6 8HD, UK \\ b School of Psychological Sciences, University of Manchester, Oxford Road, Manchester M13 9PL, UK
}

\section{A R T I C L E I N F O}

\section{Article history:}

Received 6 May 2009

Received in revised form 9 March 2010

Accepted 15 March 2010

Available online 21 March 2010

\section{Keywords:}

EEG

Laser

Anticipatory response

Laser-evoked potential

State anxiety

Pain

\begin{abstract}
A B S T R A C T
Placebo has been shown to be a powerful analgesic with corresponding reduction in the activation of the pain matrix in the brain. However, the response to placebo treatment is highly variable. It is unclear how anticipatory and pain-evoked potentials are affected by the treatment and how reproducible the response is. Laser stimulation was used to induce moderate pain in healthy volunteers. We induced placebo analgesia by conditioning subjects to expect pain reduction by applying a sham anaesthetic cream on one arm in conjunction with a reduced laser stimulus. Pain ratings were assessed before, during and after treatment. Using lectroencephalography (EEG) we measured anticipatory neural responses and pain-evoked potentials to laser heat to determine how expectation of analgesia affected the response to a placebo manipulation. This was a reproducibility study and as such the experimental procedure was repeated after a minimum gap of 2 weeks. Significant reductions in pain-evoked potentials were shown after treatment. The anticipatory responses did not change after treatment for the control and shamtreatment groups in the first session but were significantly lower in the repeat session relative to the first session in the sham-treatment group only. A significant correlation was found between the reduction in state anxiety in the repeat session relative to the first and the reduction in the anticipatory response in the sham-treatment group. Receiving a placebo treatment appears to cause a lasting change in the cognitive processing of pain for at least 6 weeks. This cognitive change may be facilitated by a change in state anxiety.
\end{abstract}

(ㄷ) 2010 Elsevier Ltd. All rights reserved.

\section{Introduction}

Expectation of treatment effect is crucial in mediating the placebo component of a therapy. Conditioning-like procedures are often used in placebo paradigms to induce expectations of clinical effect which in turn can cause decreases in pain ratings and neurophysiological responses such as decreasing the N2 and P2 components of laser-evoked potentials (LEPs) (Colloca et al., 2008; Wager, Matre, \& Casey, 2006; Watson, El-Deredy, Vogt, \& Jones, 2007). In such paradigms removing the expectation of relief results in the loss of the placebo component of the treatment (Benedetti et al., 2003) and by altering the instructions about certain and uncertain expectations of analgesia different placebo analgesic effects can be produced (Pollo et al., 2001; Price et al., 1999; Vase, Robinson, Verne, \& Price, 2003). Treatment with a placebo can produce long lasting effects. In a comparative trial of antidepressants $72 \%$ of placebo responders in the control group, kept on placebo treatment, remained in remission for the duration of the 2 year study (Montgomery, Reimitz, \& Zivkov, 1998) whilst 73\% of the long term improvement amongst patients treated with antidepressants

\footnotetext{
* Corresponding author. Tel.: +440161 2060205

E-mail address: Debbie.morton@hotmail.co.uk (D.L. Morton).
}

is duplicated in patients taking placebo (Walach \& Maidhof, 1999).

Previous studies have used fMRI to show the activation of specific brain areas by placebo that are associated with reduced pain expectations (Craggs, Price, Verne, Perlstein, \& Robinson, 2007; Scott et al., 2007; Wager, Scott, \& Zubieta, 2007). More recent studies have identified common regions within the cingulate cortex associated with the reduced anticipation of pain both during and after placebo treatment (Watson et al., 2009). However, as fMRI has a long temporal resolution it is difficult to ascertain how far in advance of the stimulus this expectation is processed. Electroencephalography (EEG) has a much shorter temporal resolution and can be used to record the stimulus preceding negativity (SPN), an anticipatory event-related potential (ERP). The SPN reflects the anticipatory state of a subject (Brunia \& van Boxtel, 2001; Kotani et al., 2003) and can be regarded as a top-down measure of cognitive processing. It is a slow negative ERP that starts to develop a few seconds before stimulus onset. The SPN not only relates to the perceptual anticipation process but also to an emotional anticipation process (Ohgami et al., 2006; Poli, Sarlo, Bortoletto, Buodo, \& Palomba, 2007) and has been shown to predict the LEP response when subjects are confident in their beliefs about pain intensity (Brown, Seymour, Boyle, El-Deredy, \& Jones, 2008a). Previous studies have shown that the SPN is enhanced by conditions as diverse as anxiety and fear (Boecker, Baas, Kenemans, \& Verbaten, 2001) and 
reward (Kotani et al., 2003; Ohgami et al., 2006). It is suggested that the main component of the SPN results from selective anticipatory attention (Bocker, Baas, Kenemans, \& Verbaten, 2001), rather than a specific emotion per se. Behaviourally, we have shown that keeping situational cues consistent results in a reproducible placebo response predicted by dispositional optimism and state anxiety scores (Morton, Watson, El-Deredy, \& Jones, 2009). To test if the placebo reproducibility extends to the anticipatory (SPN) and the experience (N2 and $\mathrm{P} 2$ ) components of the pain-evoked potentials, subjects were asked to attend two identical experimental sessions separated by a minimum of 2 weeks. In addition the following hypotheses were tested:

(1) The sham-treatment group will have reduced SPN and LEP components post-treatment compared to the control group in both sessions. This will reflect the expectation and experience of reduced pain respectively. The expected reduction in painevoked potentials follows from the behavioral results of Morton et al. (2009) which show a reproducible drop in pain ratings over the two treatment sessions and will be correlated over the two sessions. Further, the changes in pain-evoked potentials will correlate with the personality attributes of dispositional optimism and state anxiety.

(2) There will be a correlation between anticipatory SPN and the subsequent LEPs, as shown previously by Brown et al. (2008a) and Brown, Seymour, Boyle, El-Deredy, and Jones (2008b), and this correlation will be maintained over the two treatment sessions.

\section{Methods}

\subsection{Subjects}

Sixty-seven healthy right-handed subjects with no history of pain, neurological or psychiatric disorders, and no previous experience with local anaesthetic creams took part in the study. Eleven subjects were excluded from the analysis, six subjects due to exceptionally noisy EEG and five subjects who did not return for the second experimental session, leaving a sample size of 56 . Subjects attended the laboratory on two occasions separated by between 2 and 6 weeks. Written informed consent was obtained prior to the study and the Salford and Trafford local research ethics committee approved the study in full. Subjects were randomly assigned to one of two groups: a sham-treatment group (mean age $24 \pm 4.29$, male to female ratio 10:19) and a control group (mean age $26 \pm 3.34$, male to female ratio $11: 16$ ).

\subsection{Laser heat stimuli}

$\mathrm{CO}_{2}$ laser heat pulses of $150 \mathrm{~ms}$ duration with a beam diameter of $15 \mathrm{~mm}$ were applied to the dorsal surface of one forearm. The stimulated arm was pseudorandomised between subjects but remained the same in both experimental sessions. During inter-stimulus-intervals $(10 \mathrm{~s})$ the laser was randomly moved over an area of $3 \mathrm{~cm} \times 5 \mathrm{~cm}$ to avoid skin damage, habituation or sensitisation. The subjects were asked to rate the stimuli verbally using a $0-10$ pain scale where 0 is no sensation and 4 corresponds to pain threshold (Watson, El-Deredy, Bentley, Vogt, \& Jones, 2006; Watson et al., 2007). A staircase procedure determined the laser energy that elicited a non-painful level 4 and a moderately painful level 7 rating. The procedure was repeated at least three times to ensure consistency.

\subsection{Pre-experiment questionnaires}

Participants were asked to fill in the State Trait Anxiety Inventory (STAI) (Spielberger, Gorsuch, \& Lushene, 1970) to distinguish between state and trait anxiety, and the Revised Life Orientation Test (LOT-R) (Scheier, Carver, \& Bridges, 1994) measuring dispositional optimism, both previously shown to correlate with behavioural placebo results (see Morton et al., 2009).

\subsection{Task}

Participants sat in front of a computer screen displaying a $3 \mathrm{~s}$ countdown sequence to each laser pulse. The countdown sequence consisted of a circle that changed from black to grey to white at 1-s intervals (Fig. 1). This anticipatory period allowed for the recording of the SPN for each trial as previously shown by Brown et al. (2008a,b). The 0-10 pain scale was displayed after each laser pulse and subjects were asked to rate the pain.

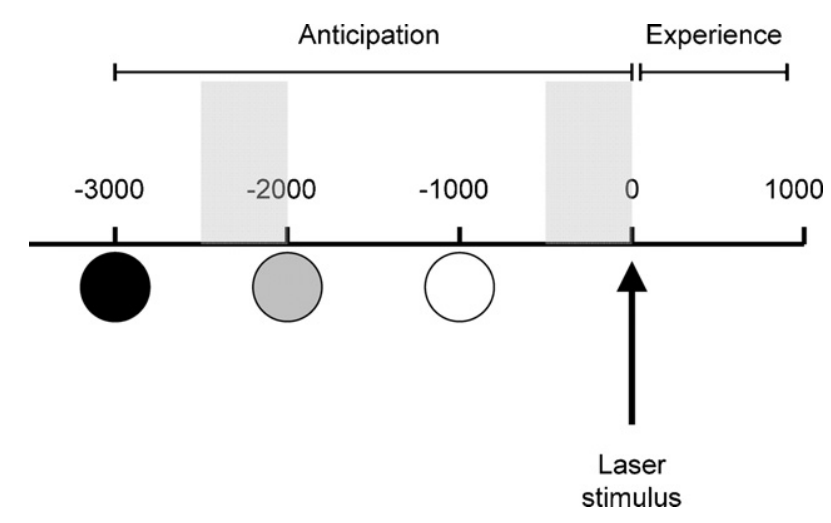

Fig. 1. A stimulus preceding negativity (SPN) was recorded $3 \mathrm{~s}$ before the pain stimulus. The countdown sequence comprised three circles that changed from black to grey to white at 1-s intervals. The grey boxes represent the two SPN windows presented here: 'early' -2500 to $-2000 \mathrm{~ms}$ and 'late' -500 to $0 \mathrm{~ms}$.

\section{Experimental design}

\subsection{Sham-treatment group}

The sham-treatment group was told that they may receive a local anaesthetic cream on their arm which would reduce the pain.

\subsubsection{Pre-treatment block}

Subjects were asked to rate 30 moderately painful level 7 pulses on the $0-10$-pain scale.

\subsubsection{Application of cream}

$2.5 \mathrm{~g}$ of aqueous cream was applied to the entire laser stimulation area on the arm, covered with an occlusive dressing and left in place for $30 \mathrm{~min}$, supposedly such that the cream would take effect. The appearance of the cream and its application procedure were similar to those used for the local anaesthetic cream EMLA. After $30 \mathrm{~min}$ the dressing was removed and the cream wiped off.

\subsubsection{Placebo treatment block}

Unbeknown to the subjects, the intensity of the laser stimulus was reduced to a non-painful level (Voudouris, Peck, \& Coleman, 1985 ) and subjects were asked to rate 30 pulses.

\subsubsection{Post-treatment block}

Unbeknown to the subjects, the laser energy was returned to the moderately painful level 7 exactly as in the pre-treatment block.

\subsection{Control group}

The same procedure was followed for subjects in the control group except they were explicitly told that an inactive cream would be applied to their arm and that the laser stimulus would be turned down to a non-painful level during the treatment block and turned back up again in the post-treatment block (Montgomery \& Kirsch, 1997). This removed any expectation of cream pain reduction.

\subsection{Electroencephalographic (EEG) recordings and data processing}

Continuous EEG was recorded from 61 scalp electrodes placed on an elasticised cap (easycap.de) according to the extended 10-20 system using Synamps (Neuroscan) and referenced to the right earlobe. Bandpass filters were set at $\mathrm{DC}-70 \mathrm{~Hz}$, with a sampling rate of $500 \mathrm{~Hz}$ and a gain of 500 . The vertical and horizontal electrooculograms (EOG) were measured for off-line reduction of blink and eye-movement artefacts. 
Table 1

Mean (and standard deviation) of pain ratings of the control and sham-treatment groups pre- and post-treatment in both experimental sessions.

\begin{tabular}{|c|c|c|c|c|c|}
\hline & & \multicolumn{2}{|l|}{ Control } & \multicolumn{2}{|c|}{ Sham-treatment } \\
\hline & & Pre-treatment & Post-treatment & Pre-treatment & Post-treatment \\
\hline First session & Mean pain rating (SD) & $6.07(0.58)$ & $5.83(0.72)^{*}$ & $6.32(0.50)$ & $4.98(1.49)^{*}$ \\
\hline Repeat session & Mean pain rating (SD) & $6.16(0.61)$ & $5.63(1.01)^{* *}$ & $6.24(0.51)$ & $4.66(1.58)^{* *}$ \\
\hline
\end{tabular}

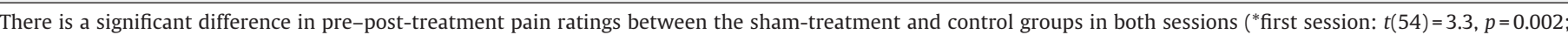
** repeat session $t(54)=2.49, p=0.02)$.

Table 2

Group $\times$ session $\times$ block ANOVA results for pain ratings, the LEP and late SPN.

\begin{tabular}{|c|c|c|c|}
\hline & Pain ratings & LEP & Late SPN \\
\hline Block & {$[F(1,54)=29.584 ; p<0.001]^{*}$} & {$[F(1,54)=42.179 ; p<0.001]^{*}$} & {$[F(1,54)=2.129 ; p=0.15]$} \\
\hline Session & {$[F(1,54)=5.683 ; p=0.02]^{*}$} & {$[F(1,54)=0.945 ; p=0.34]$} & {$[F(1,54)=3.848 ; p=0.05]^{*}$} \\
\hline Block × Group & {$[F(1,54)=4.592 ; p=0.04]^{*}$} & {$[F(1,54)=7.472 ; p=0.009]^{*}$} & {$[F(1,54)=0.169 ; p=0.68]$} \\
\hline Block $\times$ Session & {$[F(1,54)=5.133 ; p=0.03]^{*}$} & {$[F(1,54)=0.999 ; p=0.32]$} & {$[F(1,54)=0.326 ; p=0.55]$} \\
\hline Session $\times$ Group & {$[F(1,54)=0.72 ; p=0.79]$} & {$[F(1,54)=0.037 ; p=0.85]$} & {$[F(1,54)=6.254 ; p=0.02]^{*}$} \\
\hline Block $\times$ Session $\times$ Group & {$[F(1,54)=0.454 ; p=0.53]$} & {$[F(1,54)=0.180 ; p=0.67]$} & {$[F(1,54)=0.004 ; p=0.95]$} \\
\hline
\end{tabular}

"Significance at the 95\% level.

EEG data were analysed using MatLab v7.1. Eye blink artefacts were removed using principle component analysis (Casarotto, Bianchi, Cerutti, Cerutti, \& Chiarenza, 2004). Continuous EEG records were split into epochs of $4500,-500 \mathrm{~ms}$ prior to the onset of the first visual cue to $1000 \mathrm{~ms}$ after the laser pulse at time $0 \mathrm{~ms}$. For the SPN analysis, epochs were baseline corrected $500 \mathrm{~ms}$ before the onset of the first visual cue (i.e. between -3500 and $-3000 \mathrm{~ms}$ ) whereas for the LEPs analysis epochs were corrected $100 \mathrm{~ms}$ before laser onset (i.e. -100 to $0 \mathrm{~ms}$ ). Data were re-referenced to the average of the scalp electrodes.

Two $500 \mathrm{~ms}$ anticipatory periods were chosen for the SPN analysis in accordance with Brown et al. (2008a,b). An early SPN period averaging the time points over -2500 to $-2000 \mathrm{~ms}$ and a late period averaging the time points in the duration -500 to $0 \mathrm{~ms}$. The early period was the earliest part of the anticipatory response that could be measured without interference from the visual-evoked responses to the cue, and the late period measured anticipation immediately before the laser pulse. Statistical analyses on the SPN were carried out on electrodes $\mathrm{F} 3$ for the early period and $\mathrm{CPz}$ for the late period, corresponding to previously reported maximal topography of the early and late anticipatory responses respectively (Brown et al., 2008b).

N2-P2 peaks and latencies were identified from the global field power and extracted from small time windows around those latencies for each individual. The peak N2-P2 component of the LEP was measured at $\mathrm{Cz}$. The $\mathrm{N} 2$ peak was determined as the largest negative peak within a $250-350 \mathrm{~ms}$ range and the $\mathrm{P} 2$ the maximum positive peak in the $350-450 \mathrm{~ms}$ range.

\subsection{Statistical analysis}

Repeated measures ANOVAs tested the difference between preand post-treatment in subjective pain ratings, early and late SPN and LEPs in the treatment and control groups over the two test sessions (i.e. group $\times$ session $\times$ block). Correlation analysis was used to investigate the relationship between the behavioural and personality data and the EEG results. For all correlations significantly different from zero the maximum value of Cook's distance was evaluated (Cook, 1979). Cook's distance is a commonly used estimate of the influence of a data point. Values greater than one indicate large outliers which may have distorted the estimated correlation.

\section{Results}

\subsection{Behavioural results}

From Table 1 it is clear that the average pain ratings are lower post-treatment for both the control and sham-treatment groups but, as previously reported in Morton et al. (2009), the drop in reported pain is significantly larger for the sham-treatment group in both sessions (first session: $t_{54}=3.3, p=0.002$; repeat session: $t_{54}=2.49, p=0.02$ ). The ANOVA results in Table 2 show that the difference between the groups is similar in both sessions since there is no group $\times$ session interaction. The significant block $\times$ session effect shows that across both groups the decrease in reported pain is larger in the repeat session.

\subsection{Late SPN}

There were no significant patterns in the early SPN data and so we focus on the results for the late SPN. The average late SPN amplitudes shown in Fig. 2 did not support our hypothesis that the sham-treatment group would have a lower post-treatment anticipatory related potential compared to the control group. Rather, the standout result from Fig. 2 is that the average late SPN amplitude was significantly reduced in the repeat session for the sham-treatment group relative to the control group for both pretreatment $\left(t_{54}=-3.04, p=0.004\right)$ and post-treatment $\left(t_{54}=-2.33\right.$, $p=0.02$ ). Plots of the average late SPN amplitude against time in Fig. 3 clearly illustrate the difference between the sessions for the sham-treatment group.

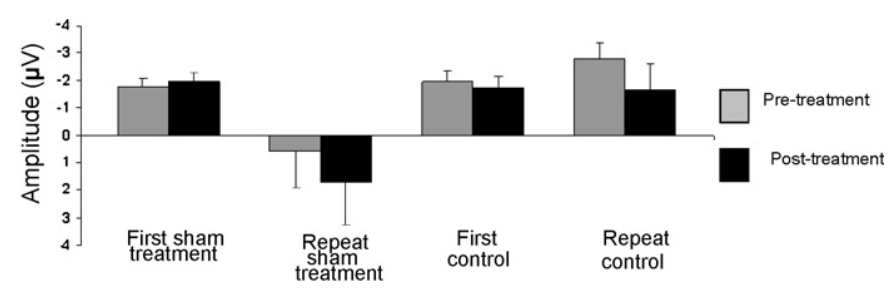

Fig. 2. The mean of the late SPN amplitudes at $\mathrm{CPz}$ with $\mathrm{SE}$ bars in the shamtreatment and control groups in both sessions, respectively. The SPN plots at electrode $\mathrm{CPz}$ where activity was maximal are shown post-treatment for the shamtreatment group in Fig. 3. 
First Session

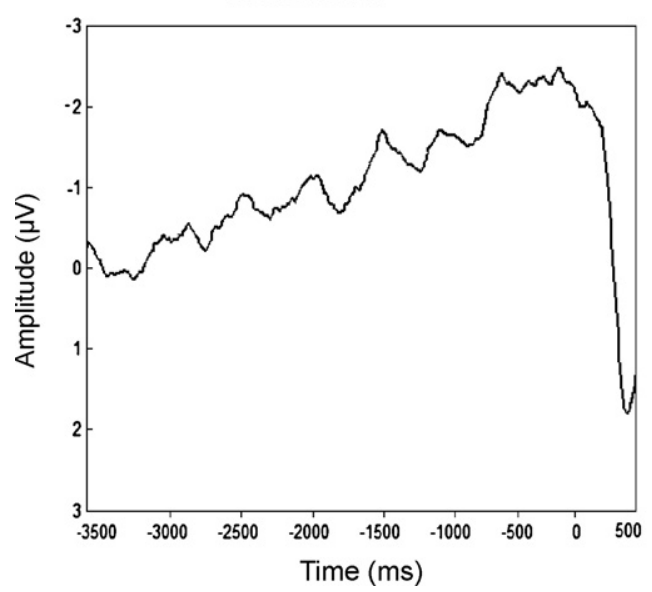

Repeat Session

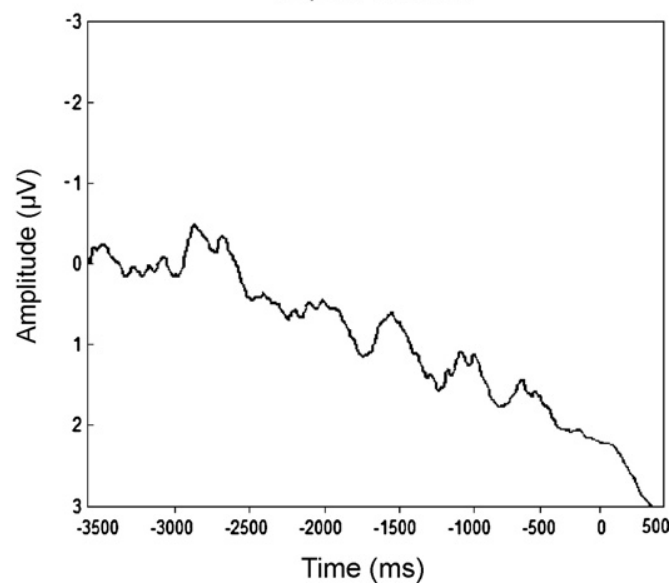

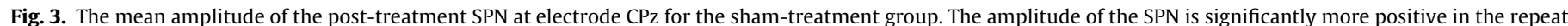
session compared to the first session $(p=0.04)$.

These findings are supported by the ANOVA results reported in Table 2 which show a highly significant session $\times$ group interaction. The fact that there is no group $\times$ block interaction again indicates that both groups had similar anticipatory responses preand post-treatment in the first session contrary to our hypothesis.

\section{3. $L E P$}

Fig. 4 shows that the sham-treatment group had a significant reduction in LEP amplitude between pre- and post-treatment in both sessions (first session $t_{54}=2.50, p=0.015$; repeat session $t_{54}=3.08, p=0.004$ ). This supports our hypothesis of a significant reduction in the LEP post-treatment in the sham-treated group. The reduction in amplitude was seen in both the N2 and the P2 components as in Colloca et al. (2008). The amplitudes of the N2-P2 in the pre- and post-treatment blocks for the sham-treatment group are illustrated in Fig. 5. The ANOVA in Table 2 identifies highly significant block and block $\times$ group interaction effects in support of this visual evidence.

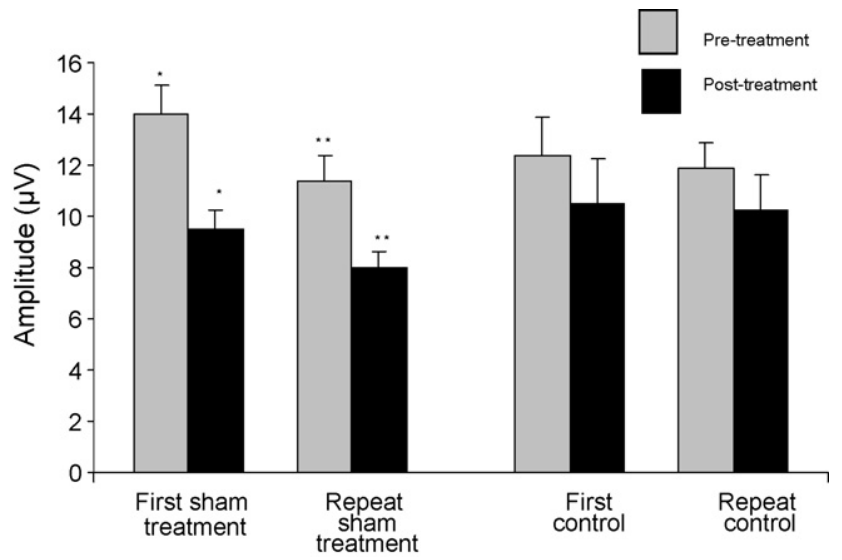

Fig. 4. Mean amplitude of the N2-P2 $(\mu \mathrm{V})$ at $\mathrm{Cz}$ for both groups and sessions with SE bars. There is a significant difference between pre- and post-treatment for the sham-treatment group in both sessions $\left({ }^{*}\right.$ Session $1 t_{54}=2.50, p=0.015$; ${ }^{* *}$ Session 2 $t_{54}=3.08, p=0.004$ ) but not for the control group.
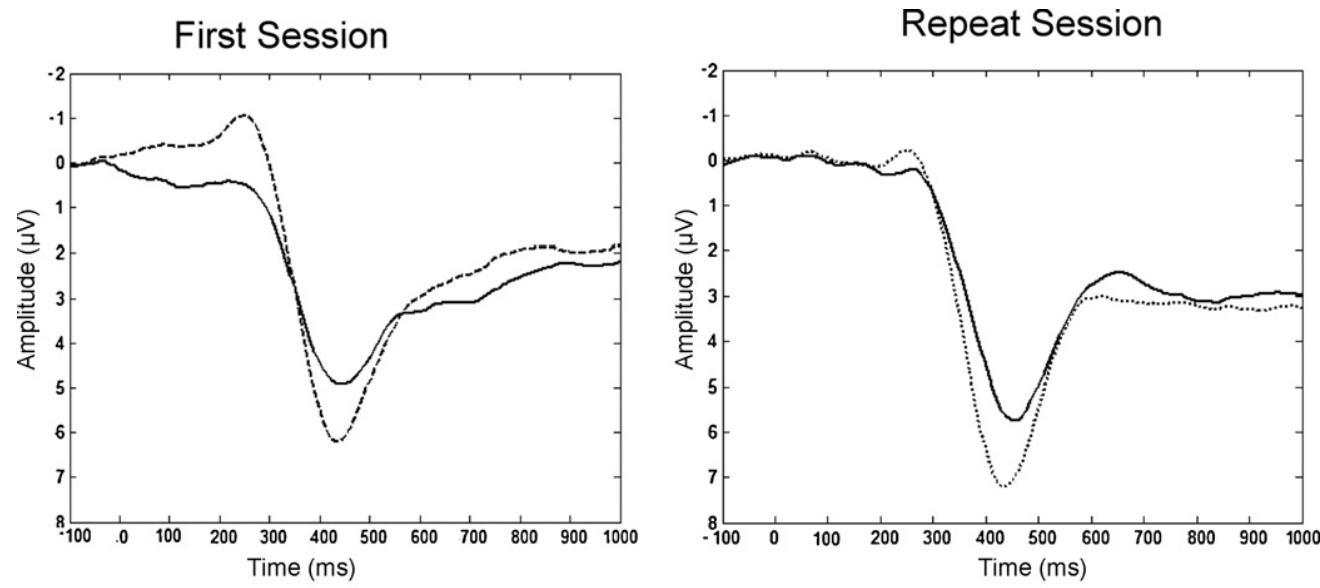

\section{......... Pre-treatment \\ Post-treatment}

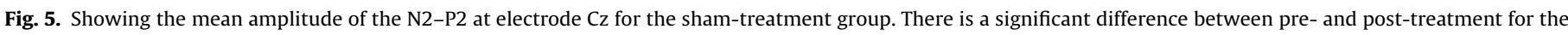
sham-treatment group in both sessions $\left({ }^{*}\right.$ Session $1 t_{54}=2.50, p=0.015 ;{ }^{*}$ Repeat session $t_{54}=3.08, p=0.004$ ). 
Table 3

Correlation analysis for the change in pain ratings, the LEP and late SPN (pre-post-treatment) in each of the two sessions for the sham-treatment group only.

\begin{tabular}{|c|c|c|}
\hline & First session & Repeat session \\
\hline Change in pain rating correlated with change in late SPN & $r=0.25(p=0.20)$ & $r=0.22(p=0.25)$ \\
\hline Change in pain rating correlated with change in LEP & $r=0.40(p=0.03 ; C=0.98)^{*}$ & $r=0.41(p=0.03 ; C=0.62)^{*}$ \\
\hline Change in LEP correlated with change in late SPN & $r=-0.42(p=0.02 ; C=0.10)^{*}$ & $r=-0.08(p=0.69)$ \\
\hline
\end{tabular}

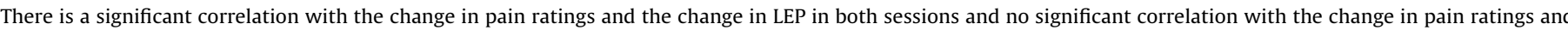

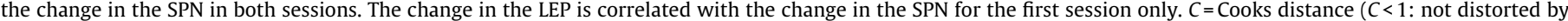
outliers).

"Significance at the $95 \%$ level.

Table 4

Correlation analysis for the change in pain ratings, the LEP and late SPN (pre-posttreatment) between the two sessions for the sham-treatment group only.

\begin{tabular}{ll}
\hline & Correlation between first and repeat session \\
\hline Change in pain rating & $r=0.74(p<0.0001 ; C=0.92)^{*}$ \\
Change in late SPN amplitude & $r=0.19(p=0.31)$ \\
Change in LEP amplitude & $r=0.41(p=0.03 ; C=0.20)^{*}$
\end{tabular}

Both the change in pain rating and change in LEP amplitude are significantly correlated between the two sessions whilst the change in the late SPN amplitude is not. $C=$ Cooks distance $(C<1$ : not distorted by outliers).

Significance at the $95 \%$ level.

\subsection{Correlation analysis}

All results discussed here are for the sham-treatment group as no significant interactions were identified for the control group.

\subsubsection{Pain ratings and EEG results-correlations over sessions}

The correlation between the change in pain ratings, anticipatory SPN and LEP amplitude from pre- to post-treatment is shown in Table 3 for both sessions.

There was a significant positive correlation between the change in pain ratings (pre-post-treatment) and the change in the LEP amplitude (pre-post-treatment) for both sessions. There was no significant relationship between the change in pain ratings (pre-post-treatment) and the change in the anticipatory SPN (pre-post-treatment) but there was a significant negative correlation between the change in the LEP amplitude (pre-post-treatment) and the change in the anticipatory SPN (pre-post-treatment) for the first session only.

\subsubsection{Pain ratings and EEG results-correlations between sessions}

Table 4 shows the correlation between the change in pain rating to the sham-treatment over the two sessions (first session, pre-treatment pain rating-post-treatment pain rating) - (repeat session, pre-treatment pain rating-post-treatment pain rating). As previously shown in Morton et al. (2009) the change in pain rating was highly reproducible and it is worth nothing that the equivalent correlation for the control group was non-significant $(p=0.92)$. The change in LEP amplitude was also found to be significantly corre- lated from the first session to the repeat session supporting our hypothesis that the physiological response will be repeatable over the two sessions. However, the hypothesis was not supported by the results on the SPN amplitude as there was no significant correlation between the change in SPN amplitude between the two sessions.

\subsubsection{The change between pre- and post-treatment and the psychological questionnaire scores}

Table 5 shows the correlation between the questionnaire scores and the changes in pain ratings, SPN amplitudes and LEP amplitudes (pre-post-treatment) in the first and repeat sessions. It can be seen that the reduction in pain ratings after treatment is significantly correlated with the state anxiety and LOT-R score in the repeat session only.

\subsubsection{The change between sessions and the psychological questionnaire scores}

Table 6 shows the correlation between the changes in pain ratings, SPN amplitudes and LEP amplitudes, over the two sessions, i.e. (first session, pre-treatment-post-treatment)-(repeat session, pretreatment-post-treatment) and the change in state anxiety over the two sessions (first session state anxiety score-repeat session state anxiety score) and the average LOT-R score over the two sessions. It can be seen that a reduction in state anxiety between the two sessions was associated with a decrease in negativity of the SPN but did not affect the LEP amplitude significantly. Meanwhile optimism can be seen to have a significant relationship with the change in pain ratings, the SPN amplitude and the LEP amplitude over the two sessions.

\section{Discussion}

In this study we investigated behavioural and electrophysiological changes due to two sessions of placebo treatment separated by 2-6 weeks. We have shown that there is a reproducible reduction in subjective pain rating and in the corresponding laserevoked potentials (N2-P2) over the two sessions. The anticipatory response, measured by the stimulus preceding negativity (SPN), was not reduced post-treatment in either session as was hypothe-

Table 5

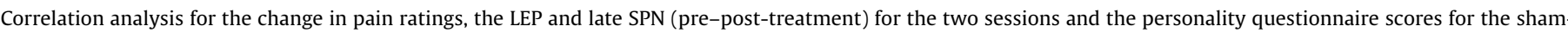
treatment group only.

\begin{tabular}{|c|c|c|}
\hline & State anxiety score & LOT-R score \\
\hline \multicolumn{3}{|l|}{ First session } \\
\hline Change in pain rating (pre-post-treatment) & $r=-0.02(p=0.93)$ & $r=0.18(p=0.28)$ \\
\hline Change in late SPN (pre-post-treatment) & $r=-0.11(p=0.50)$ & $r=-0.11(p=0.48)$ \\
\hline Change in LEP amplitudes (pre-post-treatment) & $r=-0.23(p=0.17)$ & $r=0.04(p=0.84)$ \\
\hline \multicolumn{3}{|l|}{ Repeat session } \\
\hline Change in pain rating (pre-post-treatment) & $r=-0.40(p=0.04 ; C=0.24)^{*}$ & $r=0.46(p=0.02 ; C=0.36)^{*}$ \\
\hline Change in late SPN (pre-post-treatment) & $r=0.30(p=0.13)$ & $r=0.29(p=0.14)$ \\
\hline Change in LEP amplitudes (pre-post-treatment) & $r=0.01(p=0.97)$ & $r=0.23(p=0.16)$ \\
\hline
\end{tabular}

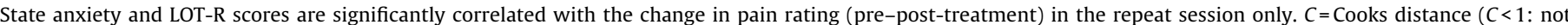
distorted by outliers).

Significance at the 95\% level. 
Table 6

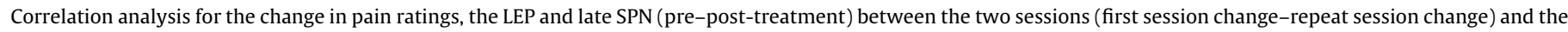
personality questionnaire scores for the sham-treatment group only.

\begin{tabular}{|c|c|c|}
\hline & Change in state anxiety (first-repeat) & Average LOT-R Score \\
\hline Change in pain rating (first-repeat) & $r=-0.16(p=0.41)$ & $r=-0.47(p=0.01 ; C=0.16)^{*}$ \\
\hline Change in late SPN (first-repeat) & $r=0.38(p=0.04 ; C=0.09)^{*}$ & $r=0.39(p=0.03 ; C=0.14)^{*}$ \\
\hline Change in LEP amplitudes (first-repeat) & $r=-0.32(p=0.09)$ & $r=-0.40(p=0.03 ; C=0.21)^{*}$ \\
\hline
\end{tabular}

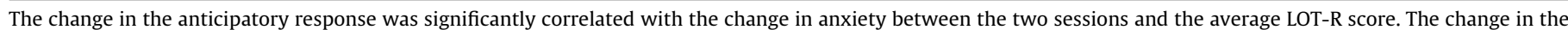
LEP was significantly correlated with the average LOT-R score only. $C=$ Cooks distance ( $C<1$ : not distorted by outliers).

Significance at the $95 \%$ level.

sised. Instead, the repeat session SPN was significantly reduced in the treatment group both pre- and post-treatment (Figs. 2 and 3), compared to the control group. This suggests that receiving the sham-treatment in the first session had an enduring cognitive effect on the way the repeat session was approached.

In the sham-treatment group we see a relationship between pain ratings and the LEP amplitude in both sessions. The relationship between the LEP and SPN amplitudes seen in the first session breaks down in the repeat session. This indicates that although the response to the treatment was reproducible, the cognitive pathway by which it was reproduced alters in the repeat session.

\subsection{The reduction in the SPN and LEP components post-treatment}

Whilst the LEP of the sham-treatment group was modulated by the treatment in both sessions and correlated over the two treatment sessions our results show that the SPN of the sham-treatment group was not. In the first session, both the sham-treatment and control group had similar SPN amplitudes pre- and post-treatment. One might argue that the laser-induced pain produced similar levels of fear and anxiety for both groups before and after treatment. This result would seem to disagree with previous fMRI data showing a lower anticipatory component post-treatment (Craggs et al., 2007; Scott et al., 2007; Wager et al., 2007). However, the anticipatory SPN is also known to increase in amplitude during paradigms inducing reward (Kotani et al., 2003; Ohgami et al., 2006). As the placebo response is thought to be modulated by reward (Faria, Fredrikson, \& Furmark, 2008; Lidstone, De La Fuente-Fernandez, \& Stoessl, 2005; Scott et al., 2007, 2008) we speculate that the increase in the component of the SPN coding for the reward of decreased pain after treatment may mask the decrease in the component of the SPN coding for fear and anxiety.

In the repeat session our results show a significant difference in the SPN of the sham-treatment group compared to the control group before and after the treatment. In the sham-treatment group, the pre- and post-treatment amplitude of the SPN was significantly lower compared to the equivalent blocks in the first session to the point where the SPN disappears. As this change in SPN amplitude was not seen in the control group it cannot be due to habituation. Instead it is indicative of lasting changes in the cognitive processing of pain being caused by a single episode of placebo treatment.

\subsection{The correlation between the changes in pain-evoked potentials and the personality attributes of dispositional optimism and state anxiety}

A correlation was found in the sham-treatment group between the change in the anticipatory SPN from the first to the repeat session and optimism (Table 6) suggesting optimists anticipate receiving a treatment prior to starting the repeat session. This fits with our behavioural results which suggested that optimists, having experienced a 'treatment' in the first session expect to receive the treatment again in the repeat session (Morton et al., 2009) We suggest that it is the expectation of treatment (driven by optimism) that reduces the emotional saliency of the stimulus in the repeat session and thus reduces state anxiety. This appears to be supported by the significant correlation seen in the sham-treatment group between the decrease in negativity of the SPN between the first and repeat sessions and the reduction in state anxiety between the two sessions (Table 6). Being able to predict upcoming painful events modulates the extent to which the stimuli will cause stress and anxiety (Carlsson et al., 2006). We speculate that expecting to receive the treatment in the repeat session lowers state anxiety prior to receiving treatment in the repeat session.

In comparison to the results seen in the sham-treatment group the control groups' pre-treatment SPN increased from the first session to the repeat session. This implies that not receiving a treatment caused the emotional saliency of the laser stimuli to increase as subjects became familiar with the procedure.

In agreement with our previous behavioural results a positive relationship was found between the change in the LEP over the two sessions and scores of dispositional optimism (Morton et al., 2009). This result shows that the higher a subjects' score of optimism the larger the change in the LEP (pre-post-treatment) becomes in the repeat session compared to the first. This suggests that although the placebo effects in a single session cannot be predicted by optimism, the maintenance of the response to the sham-treatment can be.

\subsection{The correlation between anticipatory SPN and the subsequent LEPs, maintenance over the two treatment sessions}

Our second hypothesis focused on the relationship between pain ratings, the LEP and the SPN. As with the work of Brown et al. (2008a,b), in the first experimental session the change in pain ratings (pre-post-treatment) were correlated with the change in the LEP amplitude (pre-post-treatment) which in turn was correlated with the change in the SPN amplitude (pre-post-treatment). This suggests that in the first session, participants in the shamtreatment group were anticipating reduced pain post-treatment. However, whilst the changes in pain ratings were still significantly correlated with the change in the LEP amplitude in the repeat session, the relationship between the change in LEP and SPN (pre-post-treatment) broke down. Referring back to the results of Brown et al. (2008a,b) showing that the SPN amplitude predicts the LEP when subjects are confident in their predictions about the pain intensity, our data suggests that receiving a treatment in the first session causes subjects to become less confident in their belief about the pain intensity during the repeat session. However, our previous conclusions (Section 5.1) lead us to suggest that the break down of the relationship between the SPN and LEP may simply due to a decrease in the emotional saliency of the stimulus. From these results we propose that the SPN should be viewed as reflecting attention to time with negative or positive emotional valance rather than simply the anticipation to pain.

\subsection{Implications}

The finding of a significant reduction in the anticipatory SPN amplitude in the repeat session (relative to the first session) in the sham-treatment group offers us insight into how receiving a 
placebo treatment can cognitively alter the future anticipation of pain. This finding is an especially important result for any longitudinal analgesic clinical trial that uses a placebo. It suggests that the action of receiving 'treatment' causes the anticipation of pain to become less salient on the subsequent occasions the 'treatment' is administered. Thus, the subject is able to cope better with the thought of impending pain. Our results demonstrate the significant lasting cognitive change that can be caused by administering a single treatment and offers an explanation to the phenomena of higher placebo response rates in longer duration clinical trials (Dworkin, Katz, \& Gitlin, 2005; Walach, Sadaghiani, Dehm, \& Bierman, 2005). It is important to recognise that this cognitive change is not only relevant to the use of placebo but will apply to any type of therapy administered and may have an impact upon the long term treatment of patients.

Although the current study does not have an equal male to female ratio in either group the similarities in the pre-treatment pain ratings and LEPs for the control and placebo groups in both sessions suggest that there is no effect of gender or menstrual phase on pain perception.

In conclusion our data shows a cognitive change in processing that may be mediated by the reduction in state anxiety seen between the two treatments. However, further work is needed to firmly establish a relationship between state anxiety and the decrease in the anticipatory response including the direction of this relationship.

\section{References}

Benedetti, F., Maggi, G., Lopiano, L., Lanotte, M., Rainero, I., Vighetti, S., et al. (2003). Open versus hidden medical treatments: The patient's knowledge about a therapy affects the therapy outcome. Prevention and Treatment, 6, 81-84.

Bocker, K. B. E., Baas, J. M. P., Kenemans, J. L., \& Verbaten, M. N. (2001). Stimuluspreceding negativity induced by fear: A manifestation of affective anticipation. International Journal of Psychophysiology, 43, 77-90.

Boecker, K. B. E., Baas, J. M. P., Kenemans, J. L., \& Verbaten, M. N. (2001). Stimuluspreceding negativity induced by fear: A manifestation of affective anticipation. International Journal of Psychophysiology, 43, 77-90.

Brown, C. A., Seymour, B., Boyle, Y., El-Deredy, W., \& Jones, A. K. P. (2008). Modulation of pain ratings by expectation and uncertainty: Behavioral characteristics and anticipatory neural correlates. Pain, 135, 240-250.

Brown, C. A., Seymour, B., El-Deredy, W., \& Jones, A. K. P. (2008). Confidence in beliefs about pain predicts expectancy effects on pain perception and anticipatory processing in right anterior insula. Pain, 139, 324-332.

Brunia, C. H. M., \& van Boxtel, G. J. M. (2001). Wait and see. International Journal of Psychophysiology, 43, 59-75.

Carlsson, K., Andersson, J., Petrovic, P., Petersson, K. M., Ohman, A., \& Ingvar, M. (2006). Predictability modulates the affective and sensory-discriminative neural processing of pain. Neurolmage, 32, 1804-1814.

Casarotto, S., Bianchi, A., Cerutti, S., Cerutti, S., \& Chiarenza, G. A. (2004). Principal component analysis for reduction of ocular artefacts in event-related potentials of normal and dyslexic children. Clinical Neurophysiology, 115, 609-619.

Colloca, L., Tinazzi, M., Recchia, S., Le Pera, D., Fiaschi, A., Benedetti, F., et al. (2008). Learning potentiates neurophysiological and behavioral placebo analgesic responses. Pain, 139(2), 306-314.

Cook, R. D. (1979). Influential observations in linear regression. Journal of the American Statistical Association, 74, 169-174.

Craggs, J. G., Price, D. D., Verne, G. N., Perlstein, W. M., \& Robinson, M. M. (2007) Functional brain interactions that serve cognitive-affective processing during pain and placebo analgesia. NeuroImage, 38, 720-729.
Dworkin, R. H., Katz, J., \& Gitlin, M. J. (2005). Placebo response in clinical trials of depression and its implications for research on chronic neuropathic pain. Neurology, 65, S7-19.

Faria, V., Fredrikson, M., \& Furmark, T. (2008). Imaging the placebo response: A neurofunctional review. European Neuropsychopharmacology, 18 473-485.

Kotani, Y., Kishida, S., Hiraku, S., Suda, K., Ishii, M., \& Aihara, Y. (2003). Effects of information and reward on stimulus-preceding negativity prior to feedback stimuli. Psychophysiology, 40, 818-826.

Lidstone, S. C., De La Fuente-Fernandez, R., \& Stoessl, A. J. (2005). The placebo response as a reward mechanism. Seminars in Pain Medicine, 3 , 37-42.

Montgomery, G. H., \& Kirsch, I. (1997). Classical conditioning and the placebo effect. Pain, 72, 107-113.

Montgomery, S. A., Reimitz, P. E., \& Zivkov, M. (1998). Mirtazapine versus amitriptyline in the longterm treatment of depression: A double-blind placebo-controlled study. International Clinical Psychopharmacology, 13, 63-73.

Morton, D. L., Watson, A., El-Deredy, W., \& Jones, A. K. P. (2009). Reproducibility of placebo analgesia: Effect of dispositional optimism. Pain, 146, 194-198.

Ohgami, Y., Kotani, Y., Tsukamoto, T., Omura, K., Inoue, Y., Aihara, Y., et al. (2006) Effects of monetary reward and punishment on stimulus-preceding negativity. Psychophysiology, 43, 227-236.

Poli, S., Sarlo, M., Bortoletto, M., Buodo, G., \& Palomba, D. (2007). Stimulus-preceding negativity and heart rate changes in anticipation of affective pictures. International Journal of Psychophysiology, 65, 32-39.

Pollo, A., Amanzio, M., Arslanian, A., Casadio, C., Maggi, G., \& Benedetti, F. (2001) Response expectancies in placebo analgesia and their clinical relevance. Pain 93, 77-84.

Price, D. D., Milling, L. S., Kirsch, I., Duff, A., Montgomery, G. H., \& Nicholls, S. S. (1999) An analysis of factors that contribute to the magnitude of placebo analgesia in an experimental paradigm. Pain, 83, 147-156.

Scheier, M. F., Carver, C. S., \& Bridges, M. W. (1994). Distinguishing optimism from neuroticism (and trait anxiety, self-mastery, and self-esteem): A reevaluation of the Life Orientation Test. Journal of Personality and Social Psychology, 67 1063-1078.

Scott, D. J., Stohler, C. S., Egnatuk, C. M., Wang, H., Koeppe, R. A., \& Zubieta, J. K. (2007). Individual differences in reward responding explain placebo-induced expectations and effects. Neuron, 55, 325-336.

Scott, D. J., Stohler, C. S., Egnatuk, C. M., Wang, H., Koeppe, R. A., \& Zubieta, J. K. (2008). Placebo and nocebo effects are defined by opposite opioid and dopaminergic responses. Archives of General Psychiatry, 65, 220-231.

Spielberger, C. D., Gorsuch, R. L., \& Lushene, R. D. (1970). STAI manual for the state-trait anxiety inventory. Palo Alto, CA, USA: Consulting Psychologists Press.

Vase, L., Robinson, M. E., Verne, G. N., \& Price, D. D. (2003). The contributions of suggestion, desire, and expectation to placebo effects in irritable bowel syndrome patients: An empirical investigation. Pain, 105, 17-25.

Voudouris, N. J., Peck, C. L., \& Coleman, G. (1985). Conditioned placebo responses. Journal of Personality Assessment, 47-53.

Wager, T. D., Matre, D., \& Casey, K. L. (2006). Placebo effects in laser-evoked pain potentials. Brain, Behavior, and Immunity, 10, S89-S91.

Wager, T. D., Scott, D. J., \& Zubieta, J. K. (2007). Placebo effects on human \{micro\}opioid activity during pain. Proceedings of the National Academy of Sciences of the United States of America, 26, 104-107.

Walach, H., \& Maidhof, C. (1999). Is the placebo effect dependent on time? A metaanalysis. In I. Kirsch (Ed.), Expectancy, experience and behaviour (pp. 321-332). Washington, DC: American Psychological Association.

Walach, H., Sadaghiani, C., Dehm, C., \& Bierman, D. (2005). The therapeutic effect of clinical trials: Understanding placebo response rates in clinical trials-A sec ondary analysis. BMC Medical Research Methodology, 5, 26.

Watson, A., El-Deredy, W., Bentley, E. E., Vogt, B. A., \& Jones, A. K. P. (2006). Categories of placebo response in the absence of site-specific expectation of analgesia. Pain $126,115-122$.

Watson, A., El-Deredy, W., Iannetti, G. D., LLoyd, D., Tracey, I., Vogt, B. A., et al. (2009) Placebo conditioning and placebo analgesia modulate a common brain network during pain anticipation and perception. Pain, 145, 24-30

Watson, A., El-Deredy, W., Vogt, B. A., \& Jones, A. K. P. (2007). Placebo analgesia is not due to compliance or habituation: EEG and behavioural evidence. Neuroreport $18,771-775$. 